\title{
On causal stochastic equations for log-stable multiplicative cascades
}

\author{
F. G. Schmitt ${ }^{1}$ and P. Chainais ${ }^{2}$ \\ 1 CNRS FRE 2816 ELICO, Wimereux Marine Station, Université des Sciences et Technologies de Lille - Lille 1, 28 av. Foch, \\ 62930 Wimereux, France \\ e-mail: francois.schmitt@univ-lille1.fr \\ 2 LIMOS UMR CNRS 6158-Université Blaise Pascal 63173 Aubière, France. \\ e-mail: pchainai@isima.fr, http://www.isima.fr/ chainais
}

Received: date / Revised version: date Version June 22, 2007

\begin{abstract}
We reformulate various versions of infinitely divisible cascades proposed in the literature using stochastic equations. This approach sheds a new light on the differences and common points of several formulations that have been recently provided by several teams. In particular, we focus on the simplification occurring when the infinitely divisible noise at the heart of such model is stable: an independently scattered random measure becomes a stable stochastic integral. In the last section we discuss the $D$-dimensional generalization.
\end{abstract}

PACS. 02.50.Ey Stochastic processes - 47.27.E- Turbulence simulation and modelling - 5.45.Df Fractals

\section{Introduction}

Multifractals have been introduced more than two decades ago in the fields of turbulence, geophysics and chaos theory. Since then, the multifractal framework has been used widely in many fields including turbulence [1,2], precipitations $[3,4]$, oceanography $[5,6]$, biology $[7-9]$, chemistry [10] astrophysics [11], finance [12-14], etc.

The main properties of multifractal models or data is to possess high variability on a wide range of spatial or temporal scales, associated to scaling intermittent fluctuations and long-range power-law correlations. Scale invariance is usually associated to a power-law spectrum (e.g. the $k^{-5 / 3}$ Kolmogorov law in fully developed turbulence) which is the signature of the absence of a characteristic scale. At large scales, scale invariance may be connected to long range dependence due to power law correlations. At small scales, scale invariance is rather interpreted as a singular behaviour (e.g., non differentiable) of the form $\left|f(x)-f\left(x_{o}\right)\right| \sim\left|x-x_{o}\right|^{h}$ connected to a high variability on small scales. It appears that these intepretations of the scale invariance property can be linked to the statistics of the multiscale fluctuations of some physical data (e.g., the velocity increments or the dissipation field in a turbulent flow). Indeed, there exists a parallel between the description of the scale invariant behaviour of a field by a set of multiscaling exponents and the description of the statistics of its fluctuations at various scales [15-17].

For a long time, research in this framework was mainly devoted to data analysis and to the estimation of some parameters, but no continuous model was proposed to re- produce the captured properties in the general case, and there were only discrete scale constructions like Mandelbrot binomial cascades [18] or cascades on wavelet trees [19]. Only recently, research has developed on the definitions and properties of versatile multifractal stochastic processes. In this framework, various recent works [20-24, 16] have presented similar objects in different ways, involving multifractal stochastic processes depending on a continuous scale parameter. These objects belong to the family of infinitely divisible cascades, a large class of multifractal scalar fields. We propose to review these presentations of infinitely divisible cascades which rely on the use of rather abstract mathematical objects such as additive stochastic measures. Then we show how a specific family of stochastic processes, namely stable processes (including Gaussian processes), connects to a related family of processes that is much more easier to describe. In this approach, we will use descriptions based on the framework of multiplicative cascades as well as the framework of stochastic integrals as proposed for the Gaussian case in 1 dimension in [22].

First, we consider cascades in 1 dimension only. We recall the main definitions and properties of infinitely divisible (ID) cascades. We also recall the definition using a stochastic integral proposed in [22] in the special Normal case. We extend the result of [22] describing explicitly how the ID framework is related to a simpler framework for Lévy stable random variables in 1 dimension. Then we show that most of our arguments are easier to understand in 1 dimension but they generalize rather naturally to $D \geq 2$ dimensions. Moreover, a nice formulation based 
on the use of a stochastic integral with respect to some $\alpha$ stable noise is given. Finally, we propose to consider separately $D$ spatial dimensions and 1 time dimension within a unique $D+1$ dimensional cascade so that causal animated scalar fields can be considered. Again a formulation using a stochastic integral with respect to some $\alpha$-stable noise is proposed that makes explicit the main properties of the resulting field: multifractality both in time and space, homogeneity, stationarity and causality.

\section{From cascades to stochastic integrals: the 1D causal case}

Examples of realizations of 1D, 2D and 3D infinitely divisible cascades are shown on figure 1 . Let us first recall the definition of a scale invariant infinitely divisible cascade.

\subsection{Definitions}

Let $G(X)$ be an infinitely divisible distribution with moment generating function $\tilde{G}(q)$ that can be written in the following form $\tilde{G}(q)=\mathbb{E}\left(e^{q X}\right)=e^{-\rho(q)}$.

In full generality, let $d m(t, r)=g(r) d t d r$ a positive measure on the time-scale half-plane $\mathcal{P}^{+}:=\mathbb{R} \times \mathbb{R}^{+}$. We will see below that scale invariance imposes the special choice $d m(t, r)=\frac{1}{r^{2}} d t d r$. Let $M$ denote an infinitely divisible (ID), additive independently scattered random measure (called an "ID random measure" in the following) distributed by $G$, and supported on the time-scale halfplane $\mathcal{P}^{+}$and associated to its so-called control measure $d m(t, r)$. For any subset $\mathcal{E}$ of $\mathcal{P}^{+}$, the random measure $M$ is such that

$$
\mathbb{E}[\exp [q M(\mathcal{E})]]=\exp [-\rho(q) m(\mathcal{E})] .
$$

For all disjoints subsets $\mathcal{E}_{1}$ and $\mathcal{E}_{2}, M\left(\mathcal{E}_{1}\right)$ and $M\left(\mathcal{E}_{2}\right)$ are independent random variables and $M\left(\mathcal{E}_{1} \cup \mathcal{E}_{2}\right)=M\left(\mathcal{E}_{1}\right)+$ $M\left(\mathcal{E}_{2}\right)$. For more information on random measures, see [25-27], and for a first use for cascades see [20].

\section{Definition 1}

For a given resolution $0<\ell \leq 1$, let $\mathcal{C}_{\ell}(t)$ the cone of influence defined below for every $t \in \mathbb{R}$. An Infinitely Divisible Cascading measure density (IDC measure density) is a family of processes $Q_{\ell}(t)$ parametrized by $\ell$ of the form

$$
Q_{\ell}(t)=\frac{\exp \left[M\left(\mathcal{C}_{\ell}(t)\right)\right]}{\mathbb{E}\left[\exp M\left(\mathcal{C}_{\ell}(t)\right)\right]} .
$$

Possible choices for distribution $G$ are the Normal distribution, Poisson distribution, compound Poisson distributions, Gamma laws, Lévy-stable laws, and infinitely many others, so that a large variety of choices is available for modelling and applications (see [28]).

Different definitions of the cone $\mathcal{C}_{\ell}(t)$ have been recently proposed in the literature. To get causal definitions, we adapt the expressions given in $[21,23,24]$, and consider only integration on positions $\left(t^{\prime}, r^{\prime}\right)$ for which $t^{\prime} \leq t$. The corresponding choices are ${ }^{1}$ :

$$
\left\{\begin{aligned}
\mathcal{C}_{\ell}^{B M}(t) & =\left\{\left(t^{\prime}, r^{\prime}\right): \ell \leq r^{\prime} \leq 1, t-r^{\prime} \leq t^{\prime} \leq t\right\} \\
& \cup\left\{\left(t^{\prime}, r^{\prime}\right): t-1 \leq t^{\prime} \leq t, r^{\prime} \geq 1\right\} \\
& \text { in }[21,23], \text { see fig. } 2(\mathrm{a}) ; \\
\mathcal{C}_{\ell}^{C R A}(t) & =\left\{\left(t^{\prime}, r^{\prime}\right): \ell \leq r^{\prime} \leq 1, t-r^{\prime} \leq t^{\prime} \leq t\right\} \\
& \text { in }[24], \text { see fig. } 2(\mathrm{~b}) .
\end{aligned}\right.
$$

In this contribution, we will focus on stable laws. Indeed, it appears that for stable cascades the ID stochastic measure $M$ may be simplified, and expressed using a stochastic integral versus a stable noise. Such a formulation was proposed by Schmitt and Marsan [20] and its properties in the Gaussian case studied in Schmitt [22]:

\section{Definition 2}

$$
\epsilon_{\lambda}(t)=\lambda^{-\sigma^{2} / 2} \exp \left(\sigma \int_{t+1-\lambda}^{t}(t+1-u)^{-1 / 2} d B(u)\right)
$$

where $\lambda$ plays the role of the inverse of a resolution as $1 / \ell$ in definition $1, d B(u)$ is a Gaussian noise and $\sigma^{2}$ is a variance parameter. We will use the notation $\tilde{Q}_{\ell}(t)=$ $\epsilon_{\lambda}(t)$ in the sequel.

Our first aim is to gather the various definitions above within consistent notations and to precisely formulate the link between approaches using respectively "multiplicative cascades" like (2) and "stochastic integrals" like (4). To bring all approaches within the same context, we will mainly use causal definitions.

\subsection{Scaling properties}

The scaling properties of IDCs in 1 dimension have been studied in Refs. [20-24,29,30]. We recall here some basic results. The measure $M$, the distribution $G$, the control measure $m$ and the geometry of the cone of influence $\mathcal{C}_{\ell}(\mathbf{x})$ control the scaling structure as well as marginal distributions of the cascade. One major property of IDCs is the following $[20,21,24]$ :

$$
\mathbb{E}\left[Q_{\ell}^{q}\right]=\exp \left[-\varphi(q) m\left(\mathcal{C}_{\ell}\right)\right]
$$

where

$$
\varphi(q)=\rho(q)-q \rho(1), \quad \varphi(1)=0
$$

for all $q$ for which $\rho(q)=-\log \tilde{G}(q)$ is defined.

We may also remark that, turning to local averages $\kappa_{r}$ over a box of size $r \geq \ell$

$$
\kappa_{r}(t)=\frac{1}{r} \int_{\left|t^{\prime}-t\right|<r / 2} Q_{\ell}\left(t^{\prime}\right) d t^{\prime}
$$

one gets:

$$
\mathbb{E} \kappa_{r}(t)^{q} \propto \exp \left[\tau(q) m\left(\mathcal{C}_{r}(t)\right)\right]
$$

1 Note that the large scale in the definition of $\mathcal{C}_{\ell}(t)$ has been arbitrarily set to 1 without loss of generality. Choosing a different large scale $L$ would simply reduce to a change of units $t \rightarrow t \cdot L, \ell \rightarrow r \cdot L$. 
$1 \mathrm{D}$

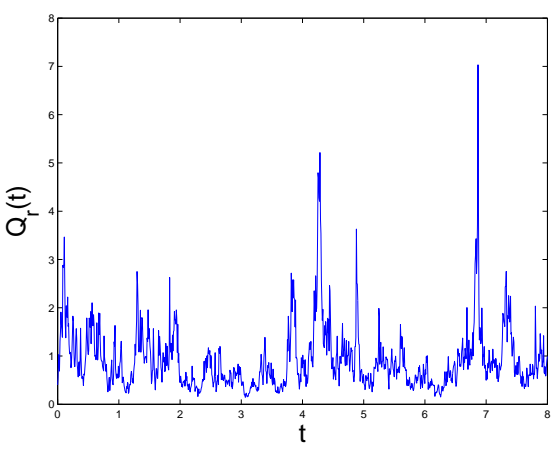

$2 \mathrm{D}$

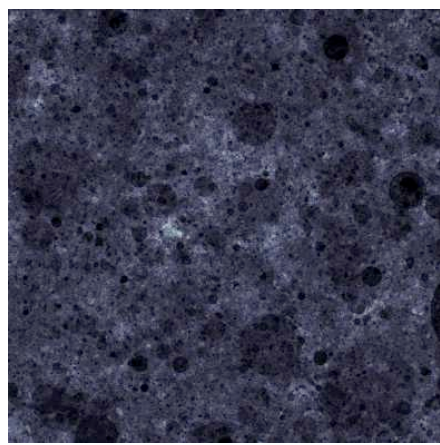

$3 \mathrm{D}$

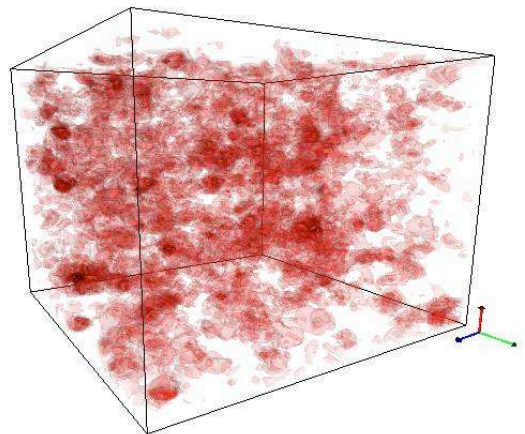

Fig. 1. Examples of realizations of infinitely divisible cascades in 1, 2 and 3 dimensions respectively.

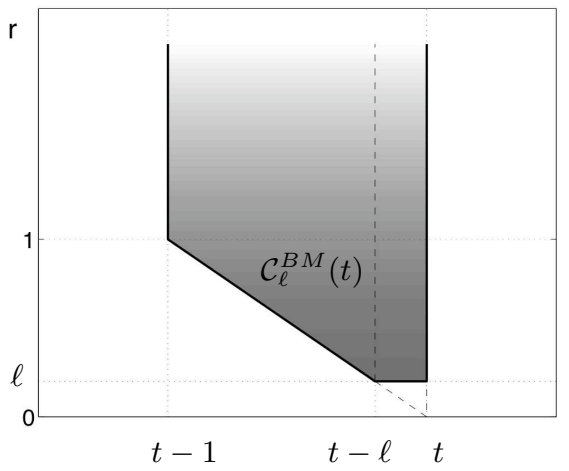

(a)

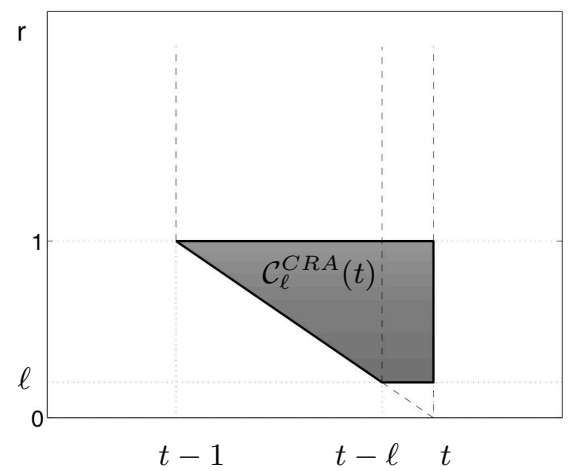

(b)

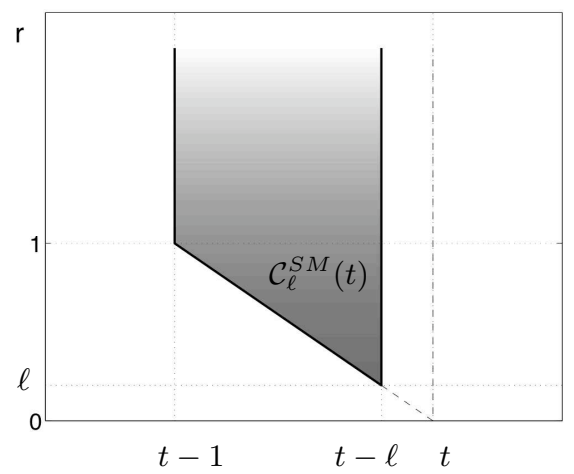

(c)

Fig. 2. Cone $\mathcal{C}_{\ell}(t)$ chosen by (a) Bacry \& Muzy [21], (b) Chainais, Riedi \& Abry [24]; (c) Schmitt and Marsan in [20] once the correspondence is established (see text).

where in general $\tau(q)=\varphi(q)$ at least within some limited range of values of $q[29,31]$. This corresponds to observables and is therefore denoted "dressed cascades" as opposed to "bare cascades" represented by $Q_{\ell}[18,3]$. We do not consider this question further here, and mainly focus on the formalism describing the construction of bare cascades.

\subsection{The Normal case}

In this section, the distribution $G$ of definition 1 is the Normal law $\mathcal{N}\left(\mu, \sigma^{2}\right)$ with average value $\mu$ and variance $\sigma^{2}$. It was shown in $[21,23]$ that an exact power law scaling is observed if (see figure 2(a)):

$$
\left\{\begin{aligned}
d m(t, r)= & \frac{d t d r}{r^{2}} \\
\mathcal{C}_{\ell}(t)= & \mathcal{C}_{\ell}^{B M}(t) \\
= & \left\{\left(t^{\prime}, r^{\prime}\right): \ell \leq r^{\prime} \leq 1, t-r^{\prime} \leq t^{\prime} \leq t\right\} \\
& \cup\left\{\left(t^{\prime}, r^{\prime}\right): t-1 \leq t^{\prime} \leq t, r^{\prime} \geq 1\right\}
\end{aligned}\right.
$$

Then, the key quantity is the control measure of the cone of influence $\mathcal{C}_{\ell}$ :

$$
\begin{aligned}
m\left(\mathcal{C}_{\ell}^{B M}(t)\right) & =\int_{\ell}^{1} \underbrace{\int_{t-r}^{t} d t^{\prime}}_{=r} \frac{d r^{\prime}}{r^{2}}+\int_{1}^{\infty} \underbrace{\int_{t-1}^{t} d t^{\prime}}_{=1} \frac{d r^{\prime}}{r^{2}} \\
& =\log (1 / \ell)+1 .
\end{aligned}
$$

The essential term that ensures a multifractal behaviour is the $\log (1 / \ell)$ term ; the last " +1 " term only ensures exact power law scaling behaviours for scales ranging from $\ell$ to 1 . In [24], only the 1 st term was used. Indeed, as $\ell \rightarrow 0$, the differences due to the choice of the cone of figure $2(\mathrm{a})$ or (b) asymptotically disappear, except around $t \simeq 1$. The contribution of the upper part $\left\{\left(t^{\prime}, r^{\prime}\right): t-1 \leq t^{\prime} \leq t, r^{\prime} \geq\right.$ $1\}$ is minor indeed.

In $[21,23]$ as in $[24,16]$, the presentations of infinitely divisible cascades are based on multiplicative cascades and their generalizations, as in definition 1. In [22], another viewpoint is proposed that evokes random walks through the use of a stochastic integral as in definition 2 . To clarify the link between these apparently different approaches, let us note that the integrals in (10) were written integrating first w.r. to $t$ and then w.r. to $r$. Inverting this order and 


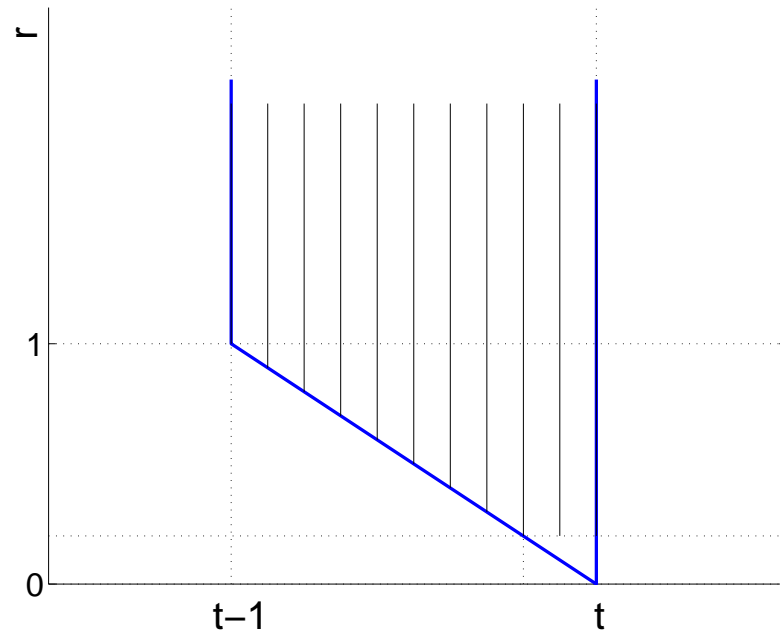

(a)

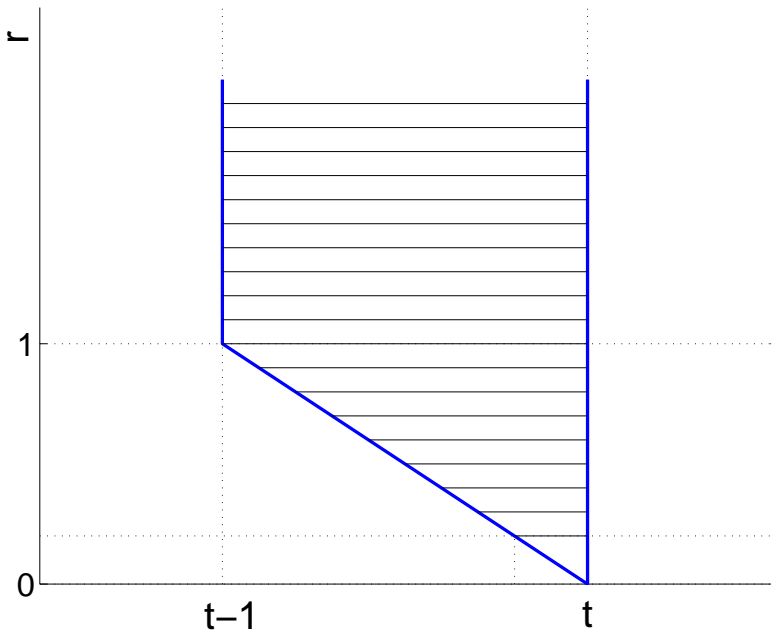

(b)

Fig. 3. (a) Integrating first w.r. to $t$, and then w.r. to $r$ : the process is described as a multiplicative cascade; (b) Integrating first w.r. to $r$, then w.r. to $t$ : the process is described as the exponential of a stochastic integral.

computing the same integrals, we obtain (see figure 3):

$$
\begin{aligned}
m\left(\mathcal{C}_{\ell}(t)\right) & =\int_{t-1}^{t-\ell} \underbrace{\int_{t-t^{\prime}}^{\infty} \frac{d r}{r^{2}}}_{\left(t-t^{\prime}\right)^{-1}} d t^{\prime}+\int_{t-\ell}^{t} \underbrace{\int_{\ell}^{\infty} \frac{d r}{r^{2}}}_{1 / \ell} d t^{\prime} \\
& =\int_{t-1}^{t-\ell}\left(t-t^{\prime}\right)^{-1} d t^{\prime}+1
\end{aligned}
$$

This writing can be used to suggest a rewriting of eq. (2) using stochastic integrals. Eq. (11) implicitly gives a decomposition of the stochastic measure $M\left(\mathcal{C}_{\ell}(t)\right)$ as a sum of successive elementary terms in time (see figure 3 ) which corresponds to a sum of independent identically distributed normal variables associated to the vertical slices on figure 3(b). This is due to the fact that $M$ is an independent and additive random measure. This way, the use of a $2 \mathrm{D}$ random measure is replaced by a $1 \mathrm{D}$ stochastic integration.

In the Normal case, one has $\rho(q)=-\mu q-\sigma^{2} \frac{q^{2}}{2}$ in (1). Thus, from (11), the random variable associated to an elementary slice $\mathcal{E}$ of width $d t^{\prime}$ around $t^{\prime}$ has mean $\propto \mu m(\mathcal{E})$ and variance $\propto \sigma^{2} m(\mathcal{E})$ where $m(\mathcal{E})=d t^{\prime} /\left(t-t^{\prime}\right)$ if $\ell \leq t-t^{\prime} \leq 1$ and $m(\mathcal{E})=1 / \ell$ if $t-t^{\prime} \leq \ell$. As a consequence, for some given instant $t$ we get the following new expression for $M\left(\mathcal{C}_{\ell}(t)\right)$, written as a stochastic integral (see also Appendix A):

$$
\begin{aligned}
M\left(\mathcal{C}_{\ell}(t)\right) \triangleq \mu \cdot m\left(\mathcal{C}_{\ell}(t)\right)+ & \sigma \int_{t-1}^{t-\ell}\left(t-t^{\prime}\right)^{-1 / 2} d B\left(t^{\prime}\right) \\
& +\sigma \int_{t-\ell}^{t} \ell^{-1 / 2} d B\left(t^{\prime}\right)
\end{aligned}
$$

where $\triangleq$ means "equality in distributions" and $m\left(\mathcal{C}_{\ell}\right)$ is given by (10) when $\mathcal{C}_{\ell}=\mathcal{C}_{\ell}^{B M}$. Equivalently, this can be written in the form

$$
M\left(\mathcal{C}_{\ell}(t)\right) \triangleq \mu \cdot m\left(\mathcal{C}_{\ell}(t)\right)+\sigma \int_{t-1}^{t} K_{\ell}\left(t-t^{\prime}\right) d B\left(t^{\prime}\right),
$$

with

$$
K_{\ell}(\tau)= \begin{cases}\tau^{-1 / 2}, & \ell \leq \tau \leq 1 \\ \ell^{-1 / 2}, & 0 \leq \tau \leq \ell\end{cases}
$$

when $\mathcal{C}_{\ell}=\mathcal{C}_{\ell}^{B M}$ as in (9). Thus, for any instant $t$, the random variable $Q_{\ell}(t)$ associated to an infinitely divisible cascade as defined by (2) can be described thanks to some Brownian motion $d B\left(t^{\prime}\right)$ by

$$
\begin{aligned}
Q_{\ell}(t) & \triangleq \frac{e^{\mu m\left(\mathcal{C}_{\ell}\right)}}{e^{\left(\mu+\sigma^{2} / 2\right) m\left(\mathcal{C}_{\ell}\right)}} \exp \left[\sigma \int_{t-1}^{t} K_{\ell}\left(t-t^{\prime}\right) d B\left(t^{\prime}\right)\right] \\
& \triangleq e^{-\frac{\sigma^{2}}{2} m\left(\mathcal{C}_{\ell}\right)} \exp \left[\sigma \int_{t-1}^{t} K_{\ell}\left(t-t^{\prime}\right) d B\left(t^{\prime}\right)\right]
\end{aligned}
$$

where $e^{-\frac{\sigma^{2}}{2} m\left(\mathcal{C}_{\ell}\right)}$ is a normalization factor.

On the way back to the formulation (4) proposed by Schmitt and Marsan in [20], let $\ell=1 / \lambda$. The change of variable $t^{\prime}=t-\frac{1}{t+1-u}$ in (4) yields:

$$
\tilde{Q}_{\ell}(t)=\epsilon_{\lambda}(t)=\ell^{\sigma^{2} / 2} \exp \left(\sigma \int_{t-1}^{t-\ell}\left(t-t^{\prime}\right)^{-1 / 2} d B\left(t^{\prime}\right)\right)
$$

where one recognizes exactly the exponential first terms of (12) with

$$
\mathcal{C}_{\ell}^{S M}(t)=\left\{\left(t^{\prime}, r^{\prime}\right): t-1 \leq t^{\prime} \leq t-\ell, r^{\prime} \geq t-t^{\prime}\right\} .
$$

Then, $m\left(\mathcal{C}_{\ell}^{S M}\right)=\log (1 / \ell)$ and the normalization factor $e^{-\sigma^{2} m\left(\mathcal{C}_{\ell}\right) / 2}$ in $(15)$ becomes $\ell^{\sigma^{2} / 2}$ in (16). The cone $\mathcal{C}_{\ell}^{S M}$ is illustrated on fig. 2(c). The difference between this cone and the cone of figure $2(\mathrm{a})$ is a thin $\operatorname{strip}\left\{\left(t^{\prime}, r^{\prime}\right): t-\ell \leq\right.$ 
$\left.t^{\prime} \leq t, r^{\prime} \geq \ell\right\}$ that becomes infinitely thin and negligible as $\ell \rightarrow 0$. We have indeed shown that this formulation is closely related to $(2)$.

Last, note that choosing the cone proposed by Chainais et al. in [24] -see figure 2(b)- yields

$$
\begin{gathered}
M\left(\mathcal{C}_{\ell}^{C R A}(t)\right) \triangleq \mu \cdot \log (1 / \ell)+\sigma \int_{t-1}^{t-\ell}\left(\frac{1}{t-t^{\prime}}-1\right)^{1 / 2} d B\left(t^{\prime}\right) \\
+\sigma \int_{t-\ell}^{t}\left(\frac{1}{\ell}-1\right)^{1 / 2} d B\left(t^{\prime}\right) .
\end{gathered}
$$

Again, the difference between (12) and (18) is sensitive for $\ell \simeq 1$ only, and asymptotically disappears as $\ell \rightarrow 0$.

It is important to note that the equalities in distribution in equations (12) to (15) are valid for one given instant $t$ only. Indeed, (15) could not be written simultaneously with the same Brownian motion for two distinct instants $t_{1} \neq t_{2}$. Therefore, $\tilde{Q}_{\ell}(t)=\epsilon_{\lambda}(t)$ in $(16)$ and $Q_{\ell}(t)$ in (15) are not identical processes. One can simply say that, for a given instant $t$, the random variables $Q_{\ell}(t)$ and $\tilde{Q}_{\ell}(t)$ are equal in distribution. Something has been "lost" from $Q_{\ell}(t)$ to $\tilde{Q}_{\ell}(t)$ : the independence of the (time, scale) stochastic measure $M$ in scale is no more present in the definition of $\epsilon_{\lambda}$ where only the integration over time remains. Given a Brownian motion $d B\left(t^{\prime}\right)$, the correlations of the process $\tilde{Q}_{\ell}(t)$ are controlled by the kernel $K_{\ell}(\tau)$. However, it was shown in [22] that the essential multifractal properties have been preserved. In particular, one recovers the characteristic property of multiplicative cascades $\operatorname{cov}\left(\log \tilde{Q}_{\ell}(0), \log \tilde{Q}_{\ell}(\tau)\right) \sim \sigma^{2} \log (1 / \tau)$. This important remark remains valid for all the generalizations considered below.

\subsection{The stable non-Gaussian case}

Previous results generalize easily to the stable case, i.e., for the choice $G=S(\alpha, \sigma, \mu, \beta)[26]$, provided the law $G$ is asymmetrical with $\beta=-1$, this condition being imposed by the fact that we need some moments of the exponential of the stable stochastic process to exist (see Schertzer and Lovejoy [3,2] and Kida [32]). Then, the function $\rho(q)$ in (1) takes the form $\rho(q)=-\mu q-\sigma^{\alpha} q^{\alpha}$ with $0<\alpha<2(\alpha \neq 1)$.

The same framework as before gives eq. (13) with a Lévy stable measure $d L_{\alpha}$ with parameter $0 \leq \alpha \leq 2$. From (11), the random variable associated to an elementary slice $\mathcal{E}$ of width $d t^{\prime}$ around $t^{\prime}$ has now mean $\propto \mu m(\mathcal{E})$ and scale parameter $\propto \sigma^{\alpha} m(\mathcal{E})$ where again $m(\mathcal{E})=d t^{\prime} /(t$ $\left.t^{\prime}\right)$ if $\ell \leq t-t^{\prime} \leq 1$ and $m(\mathcal{E})=1 / \ell$ if $t-t^{\prime} \leq \ell$. Therefore, this provides the following expression:

$$
M\left(\mathcal{C}_{\ell}(t)\right) \triangleq \mu \cdot m\left(\mathcal{C}_{\ell}(t)\right)+\sigma \int_{t-1}^{t} K_{\ell}\left(t-t^{\prime}\right) d L_{\alpha}\left(t^{\prime}\right)
$$

where the kernel is here:

$$
K_{\ell}(\tau)= \begin{cases}\tau^{-1 / \alpha}, & \ell \leq \tau \leq 1, \\ \ell^{-1 / \alpha}, & 0 \leq \tau \leq \ell .\end{cases}
$$

The choice $\alpha=2$ gives again the Gaussian case.

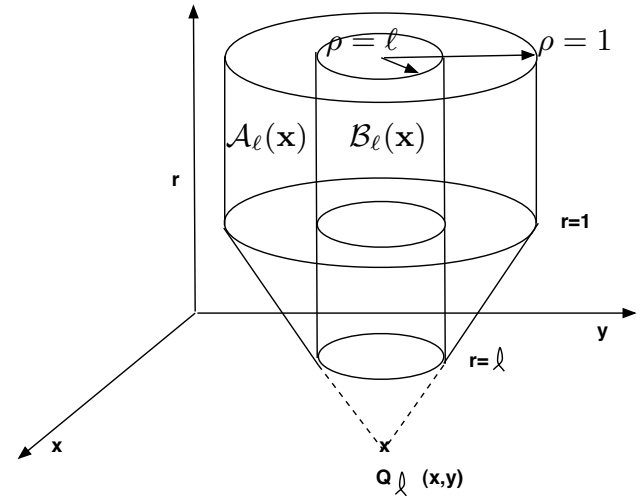

Fig. 4. Cone $\mathcal{C}_{\ell}(\mathbf{x}=(x, y))$ for $D=2$ decomposed in two parts $\mathcal{A}_{\ell}(\mathbf{x})$ and $\mathcal{B}_{\ell}(\mathbf{x})$.

\section{The 2-dimensional case}

A generalization of infinitely divisible cascades to $D$ dimensions was proposed in [16]. Examples of realizations in $2 \mathrm{D}$ and $3 \mathrm{D}$ generated using the algorithms proposed in this reference are shown on figure 1 . For pedagogical reasons, we first consider the $2 \mathrm{D}$ case in this section before going to $D$-dimensional generalization in the next section. For $D=2$ dimensions we have for $\mathbf{X} \in \mathbb{R}^{2}$

$$
d m(\mathbf{X}, r)=C(2) \frac{d r}{r^{3}} d \mathbf{X}
$$

where $C(2)$ is a constant that will be estimated below, and $\mathbf{X}=(x, y)$ is a point in the $2 \mathrm{D}(x, y)$ space. The first subsection below deals with the $2 \mathrm{D}$ spatial case and the second subsection deals with the causal case where the $y$ dimension is replaced by time $t \equiv y$.

\subsection{The two-dimensional spatial case}

In the $2 \mathrm{D}$ spatial case, the quantity $M\left(\mathcal{C}_{\ell}\right)$ results from an integral over a conical volume defined by:

$$
\begin{aligned}
\mathcal{C}_{\ell}(\mathbf{X})= & \left\{\left(\mathbf{X}^{\prime}, r^{\prime}\right): \ell \leq\left\|\mathbf{X}^{\prime}-\mathbf{X}\right\| \leq 1, r^{\prime} \geq\left\|\mathbf{X}^{\prime}-\mathbf{X}\right\|\right\} \\
& \cup\left\{\left(\mathbf{X}^{\prime}, r^{\prime}\right):\left\|\mathbf{X}^{\prime}-\mathbf{X}\right\| \leq \ell, r^{\prime} \geq \ell\right\} .
\end{aligned}
$$

as described on figure 4 . Here the cone belongs to a 3 dimensional space: 2 dimensions for the vector $\mathbf{X}=(x, y)$ and 1 dimension for the scale $r$. We must also underline that the norm $\|$.$\| in the above equation concerns the 2-$ dimensional projection of a point having a $3 D$ position $(\mathbf{X}, r)$.

The key quantity to compute is $m\left(\mathcal{C}_{\ell}(\mathbf{X})\right)$.

$$
\begin{aligned}
m\left(\mathcal{C}_{\ell}(\mathbf{X})\right)= & \iint_{\ell \leq\left\|\mathbf{X}^{\prime}-\mathbf{X}\right\| \leq 1}\left(\int_{\left\|\mathbf{X}^{\prime}-\mathbf{X}\right\|}^{\infty} \frac{C(2) d r}{r^{3}}\right) d \mathbf{X}^{\prime} \\
& +\iint_{\left\|\mathbf{X}^{\prime}-\mathbf{X}\right\| \leq \ell}\left(\int_{\ell}^{\infty} \frac{C(2) d r}{r^{3}}\right) d \mathbf{X}^{\prime}
\end{aligned}
$$


where we have again integrated w.r. to $r$ first as in (11). We then note that the integration over the conic volume can be decomposed into a sum of thin cylindrical tubes. We will therefore use below cylindrical coordinates with $\rho=$ $\left\|\mathbf{X}^{\prime}-\mathbf{X}\right\|$. Let $\mathcal{S}_{D}$ the surface of the $D$ dimensional unitary sphere (e.g. $2 \pi$ in 2 dimensions, $4 \pi$ in 3 dimensions...). This yields in 2 dimensions:

$$
\begin{aligned}
m\left(\mathcal{C}_{\ell}(\mathbf{X})\right)= & \int_{\ell}^{1}\left(\int_{\rho}^{\infty} \frac{C(2) d r}{r^{3}}\right) \mathcal{S}_{2} \rho d \rho \\
& +\int_{0}^{\ell}\left(\int_{\ell}^{\infty} \frac{C(2) d r}{r^{3}}\right) \mathcal{S}_{2} \rho d \rho \\
= & \frac{C(2) \mathcal{S}_{2}}{2}\left(\log \left(\frac{1}{\ell}\right)+\frac{1}{2}\right) \\
= & \log \left(\frac{1}{\ell}\right)+\frac{1}{2} \quad \text { if } \frac{C(2) \mathcal{S}_{2}}{2}=1 .
\end{aligned}
$$

which does not depend on $\mathbf{X}$. This last condition prescribes the choice $C(2)=1 / \pi$. Then, self-similarity is ensured in the same conditions as in dimension 1 , due to the choice of the control measure in (21).

By computing the integrals w.r. to $r$ in (23), we get from arguments similar to those used in 1 dimension (see also Appendix 2) the following expression for any given position $\mathbf{X}$ :

$$
\begin{aligned}
& M\left(\mathcal{C}_{\ell}(\mathbf{X})\right) \triangleq \mu \cdot m\left(\mathcal{C}_{\ell}\right) \\
& \quad+\sigma \iint_{\left\|\mathbf{X}^{\prime}-\mathbf{X}\right\| \leq 1} K_{\ell}^{(2)}\left(\left\|\mathbf{X}^{\prime}-\mathbf{X}\right\|\right) d L_{\alpha}\left(\mathbf{X}^{\prime}\right),
\end{aligned}
$$

where the new kernel is:

$$
K_{\ell}^{(2)}(\rho)=\left\{\begin{array}{l}
2^{-1 / \alpha} \rho^{-2 / \alpha}, \text { for } \ell \leq \rho \leq 1 \\
2^{-1 / \alpha} \ell^{-2 / \alpha}, \text { for } 0 \leq \rho \leq \ell
\end{array}\right.
$$

for $0<\alpha \leq 2$. For $\alpha<2, d L_{\alpha}$ is, as before, an $\alpha$-stable random measure with asymmetric parameter $\beta=-1$. Section 4 presents the general version of this result in $D$ dimensions.

\subsection{The 2D space-time causal case: $\mathbf{X}=(x, t)$}

It was also proposed in [16] to use multi-dimensional infinitely divisible cascades to generate time evolving processes. Here we consider the $2 D$ space-time causal case. The $y$ dimension is now considered as a time variable and denoted $t \equiv y$ so that $\mathbf{X}=(x, t)$. Our purpose is to show that the previous construction can be used to build some time evolving scalar field. The resulting field can be seen as a 1D spatial multifractal field obeying some multifractal time evolution. Causality is guaranteed by performing a stochastic integration over a cylinder analogous to the one in previous section but with the further condition $t^{\prime} \leq t$ (or $y^{\prime} \leq y$ ), see fig. 5 . The previous approach applies with only some changes due to the introduction of causality. For instance, the normalizing constant $C(2)$ becomes $2 / \pi$ in place of $1 / \pi$. The previous modifications of the stochastic

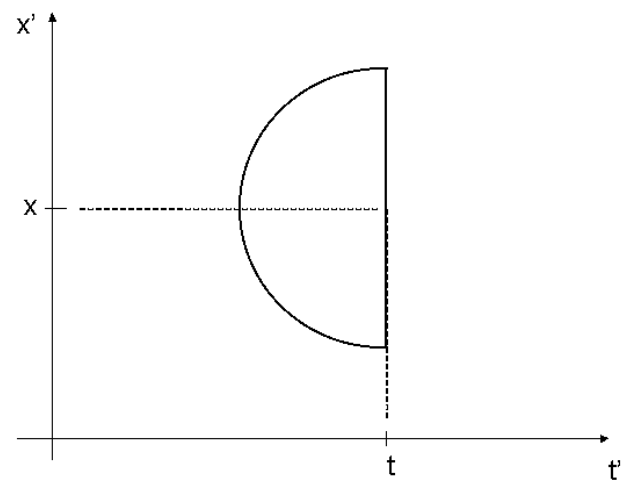

Fig. 5. Spatial domain of integration corresponding to a stable random measure for a $2 \mathrm{D}$ stable process on a space $(x, t)$.

integration for stable random measures still apply, and we obtain here the following expression for any given position $\mathbf{X}$ :

$M\left(\mathcal{C}_{\ell}(\mathbf{X})\right) \triangleq \mu \cdot m\left(\mathcal{C}_{\ell}\right)+\sigma \iint_{\mathcal{F}} K_{\ell}^{(2)}\left(\left\|\mathbf{X}^{\prime}-\mathbf{X}\right\|\right) d L_{\alpha}\left(\mathbf{X}^{\prime}\right)$,

where $\mathbf{X}=(x, t)$ and $\mathcal{F}=\left\{\mathbf{X}^{\prime}=\left(x^{\prime}, t^{\prime}\right):\left\|\mathbf{X}^{\prime}-\mathbf{X}\right\| \leq\right.$ $\left.1 ; t^{\prime} \leq t\right\}$ : see Fig 5 . The $2 \mathrm{D}$ causal kernel is still given by equation (27). Section 4 presents the general version of this result in $D$ dimensions.

Then, the origin of temporal, respectively spatial, correlations in the resulting process $\tilde{Q}_{\ell}(x, t)$ can simply be explained geometrically. The correlations are governed by the measure of the intersection of half-hyperspheres in the $(x, t)$ domain. Figure 6(a) illustrates the origin of shared information between successive values of the process at some given position, $\tilde{Q}_{\ell}\left(x, t_{1}\right)$ and $\tilde{Q}_{\ell}\left(x, t_{2}\right)$. Figure $6(\mathrm{~b})$ shows the origin of spatial correlations between $\tilde{Q}_{\ell}\left(x_{1}, t\right)$ and $\tilde{Q}_{\ell}\left(x_{2}, t\right)$ at some given instant $t$. Figure $6(\mathrm{c})$ shows the most general situation for different time instants $t_{1} \neq$ $t_{2}$ and space positions $x_{1} \neq x_{2}$.

Such a time dependent positive valued process $\tilde{Q}_{\ell}(x, t)$ exhibits a multifractal behaviour both in time and space. Moreover, it can be considered as more realistic than the approach proposed in [33-35]. Indeed, in these works, temporal correlations at large time intervals are controlled by the same quantities that control spatial correlations over small distances. This is inherent to their construction. We emphasize that there is not such a paradox in the present definition: temporal correlations over large time intervals are not dominated by spatial correlations over small distances.

\section{The D-dimensional case with $D \geq 2$}

\subsection{The spatial case}

As shown in [16], the definitions naturally extends from 1 to $D>1$ dimensions by using

$$
d m(\mathbf{X}, r)=C(D) \frac{d r}{r^{D+1}} d \mathbf{X}
$$




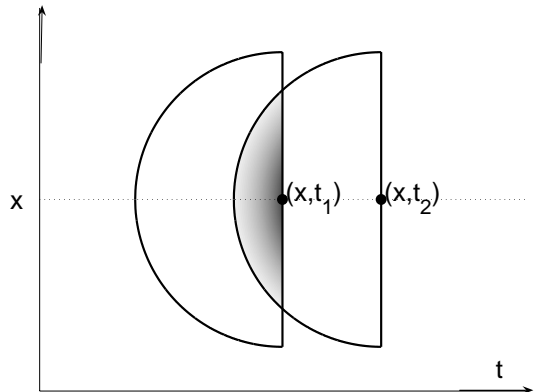

(a)

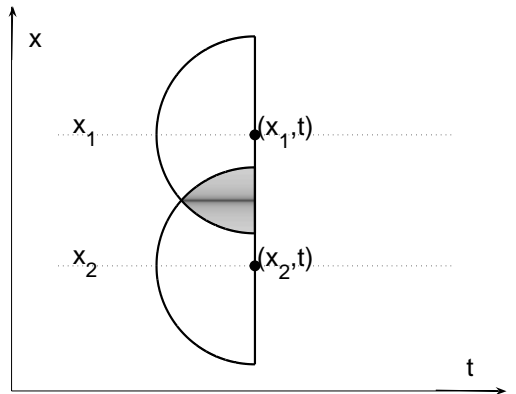

(b)

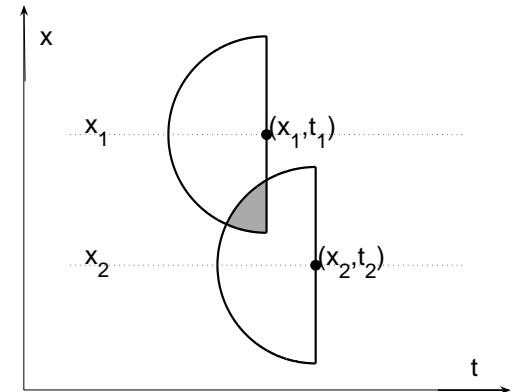

(c)

Fig. 6. Illustration of the origin of (a) temporal, respectively (b) spatial, correlations in the resulting process $\tilde{Q}_{\ell}(x, t)$. The correlations can be described in terms of the measure of the intersection of half-hyperspheres in the $(x, t)$ domain.

where $C(D)$ is a constant linked to the surface of a hypersphere of dimension $D$, and chosen to ensure the adequate scaling law.

The definition of $\mathcal{C}_{\ell}(\mathbf{X})$, the conical volume in $D$-dimension is still given by equation (22), where the cone belongs to a dimension $D+1: D$ dimensions for the vector $\mathbf{X}$ and 1 dimension for the scale $r$. As before, the norm $\|$.$\| con-$ cerns the $D$-dimensional projection of a point of position $(\mathbf{X}, r)$.

Computations similar to those of $(23)$ \& (25) performed in $D$ dimensions yield:

$$
\begin{aligned}
m\left(\mathcal{C}_{\ell}(\mathbf{x})\right)=\int_{\ell}^{1} & \left(\int_{\rho}^{\infty} \frac{C(D) d r}{r^{D+1}}\right) \mathcal{S}_{D} \rho^{D-1} d \rho \\
& +\int_{0}^{\ell}\left(\int_{\ell}^{\infty} \frac{C(D) d r}{r^{D+1}}\right) \mathcal{S}_{D} \rho^{D-1} d \rho
\end{aligned}
$$

so that $C(D)=D / \mathcal{S}_{D}=D \Gamma(D / 2) /\left(2 \pi^{D / 2}\right)$ since $\mathcal{S}_{D}=$ $2 \pi^{D / 2} / \Gamma(D / 2)$ where $\Gamma$ is the gamma function. This choice finally yields

$$
m\left(\mathcal{C}_{\ell}(\mathbf{X})\right)=\log \left(\frac{1}{\ell}\right)+\frac{1}{D}
$$

which does not depend on $\mathbf{X}$. Therefore, the previous approach for $D=2$ for an $\alpha$-stable stochastic measure generalizes to $D$ dimensions providing an expression with a polar isotropic kernel:

$$
\begin{aligned}
& M\left(\mathcal{C}_{\ell}(\mathbf{X})\right) \triangleq \mu \cdot m\left(\mathcal{C}_{\ell}\right) \\
& \quad+\sigma \iint_{\left\|\mathbf{X}^{\prime}-\mathbf{X}\right\| \leq 1} K_{\ell}^{(D)}\left(\left\|\mathbf{X}^{\prime}-\mathbf{X}\right\|\right) d L_{\alpha}\left(\mathbf{X}^{\prime}\right),
\end{aligned}
$$

where the new kernel is for $0<\alpha \leq 2$ :

$$
K_{\ell}^{(D)}(a)= \begin{cases}D^{-1 / \alpha} \rho^{-D / \alpha}, & \text { for } \ell \leq \rho \leq 1 \\ D^{-1 / \alpha} \ell^{-D / \alpha}, & \text { for } 0 \leq \rho \leq \ell .\end{cases}
$$

For $D=1$ and $D=2$, we recover the kernel described respectively by (20) and (27). As a result, we get a new description of a $D$-dimensional process $\tilde{Q}_{\ell}$ that is very similar to $Q_{\ell}(\mathbf{X})$ (see discussion in section 2.3) as defined by (2) by using a stochastic integral over the unitary disc $\left\{\mathbf{X}^{\prime}:\left\|\mathbf{X}^{\prime}-\mathbf{X}\right\| \leq 1\right\}$ (in $D$ dimension). Interestingly, this can be compared to the heuristic derivation of the $D$ dimensional stable case presented in $[3,2]$. These authors also introduced a power-law integration kernel over some volume, but the exponent was not the same: $K(\rho) \approx \rho^{-\gamma}$ with $\gamma=1-D+D / \alpha$.

An important remark concerns the simulation of logstable multiplicative cascades. They are usually rather painful to simulate because they do not belong to the family of compound Poisson cascades, see [24]. The results presented here and eq. (32) in particular give us a much more simple way to simulate not exactly identical but very similar processes. One must first simulate the independent random measure $d L_{\alpha}(\mathbf{X})$, which remains to simulate a set of i.i.d. stable variables over some regular discrete sampling of the $\mathbf{X}$ space. Then, one simply uses eq. (32) to get $M\left(\mathcal{C}_{\ell}(\mathbf{X})\right)$. Taking the exponential yields $\tilde{Q}_{\ell}(\mathbf{X})$.

Once again, it is important to note that equation (32) holds for one given position $\mathbf{X}$ only to which $d L_{\alpha}\left(\mathbf{X}^{\prime}\right)$ is indeed associated. Therefore, we emphasize that the process $\tilde{Q}_{\ell}(\mathbf{X})$ obtained by taking the exponential of (32) is not identical to $Q_{\ell}(\mathbf{X})$ as defined by (2). However, as already mentioned in section 2.3 , the essential multifractal properties are preserved.

\subsection{The causal spatio-temporal case}

We come back to the use of $D$-dimensional infinitely divisible cascades to build time evolving processes as already evoked in section 3.2 and introduced in [16]. Some "multifractal films" can be synthesized this way ${ }^{2}$. In this spirit, the same approach as above for 2-dimensional causal cascades can be easily generalized to $D$ dimensions by using $\mathbf{X}=(\mathbf{x}, t)$. Again, we will use half of a cone $\mathcal{C}_{\ell}$ as defined above: the half for which $t^{\prime} \leq t$. The resulting field can be seen as a $D$-1-dimensional multifractal field, with a time

\footnotetext{
${ }^{2}$ See http://www.isima.fr/ chainais/PUB/software.html; an example of a multifractal film, obtained by simulating a $2+1$ dimensional field and considering 2 spatial dimensions and 1 time dimension.
} 
evolution. The previous simplifications of the random integration for stable random measures still apply, and we obtain the following final expression:

$$
M\left(\mathcal{C}_{\ell}(\mathbf{X})\right) \triangleq \mu \cdot m\left(\mathcal{C}_{\ell}\right)+\sigma \iint_{\mathcal{F}} K_{\ell}^{(D)}\left(\left\|\mathbf{X}^{\prime}-\mathbf{X}\right\|\right) d L_{\alpha}\left(\mathbf{X}^{\prime}\right),
$$

where $\mathbf{X}=(\mathbf{x}, t)$ and $\mathcal{F}=\left\{\mathbf{X}^{\prime}=\left(\mathbf{x}^{\prime}, t^{\prime}\right): \|\left(\mathbf{x}^{\prime}, t^{\prime}\right)-\right.$ $\left.(\mathbf{x}, t) \| \leq 1 ; t^{\prime} \leq t\right\}$. The $D$-dim causal kernel is still given by equation (33).

The comments on figure 6 are still relevant in this general context. As a consequence, this construction inherits some nice geometrical understanding. Such a description makes explicit:

- the stationarity of the process at fixed position $\mathbf{x}_{o}$;

- a causal time evolution;

- a description in terms of a stochastic integral with respect to a stable noise $d L_{\alpha}$;

- a correspondence between this description where time and space variables are explicitely distinguished and the global description $\exp M\left(\mathcal{C}_{\ell}(\mathbf{x}, t)\right)$ where $\mathbf{x} \in \mathbb{R}^{d}$ and $t \in \mathbb{R}$ are indeed merged.

Such a construction can be compared to the fractionally integrated fields described in [3] and later extended to causal $(\mathrm{D}+1)$ space-time multifractal processes in [36]. In the latter, a Fourier approach was proposed to impose the fundamental property $\operatorname{cov}\left(\log \epsilon_{\lambda}(0), \log \epsilon_{\lambda}(\mathbf{x})\right) \sim$ $\sigma^{2} \log (1 /\|\mathbf{x}\|)$ thanks to $k^{-1}$ filtering in the Fourier domain. The resulting processes are causal, but, due to the use of a Fourier transform, they could not be synthesized in a causal manner. Moreover, such a Fourier approach is memory demanding. The definitions in terms of stochastic integrals proposed here overcome such drawbacks and provide an explicit description directly in the space-time domain.

Moreover, note that definition (34) could be formulated in the framework of Generalized Scale Invariance [37, $38]$ as well. This framework permits to introduce some controled anisotropy by replacing the classical self-similarity property in $D+1$ dimensions by a matrix operator selfsimilarity. For instance, this can be achieved by using (see equation (44) in [36])

$$
\|(\mathbf{x}, t)\|=\left(|\mathbf{x}|^{d_{e l}-D / \alpha}+|t|^{\frac{d_{e l}-D / \alpha}{1-H}}\right)^{\frac{1}{)_{e l}-D / \alpha}}
$$

where $H$ characterizes the anisotropy $(H=0$ in the isotropic case) and $d_{e l}=D+1-H$. This could be helpful to understand better how large-scale anisotropy in turbulent flows have influence at small scales. Indeed, in Kolmogorov's homogeneous turbulence framework, it is often assumed that the cascade processes "washes" the details of the large-scale flow, so that after few cascade steps, small scales become locally isotropic. In fact experimental results indicate that this may not be true, and the present approach may provide a theoretical framework to develop continuous anisotropic and scaling models.

\section{Conclusion and discussion}

In this paper, we have first given a consistent presentation of various definitions and presentations of stable cascades, a special family of infinitely divisible cascades, in 1 dimension. To this aim, we have used both the multiplicative cascade viewpoint as in $[20,21,23,24,16]$ and the stochastic integral viewpoint as in [22]. We have made explicit the differences between definitions mainly in terms of the chosen cone $\mathcal{C}_{\ell}$ when using the multiplicative cascade approach or in terms of the chosen integration domain when using the stochastic integral approach. In the latter, the integration kernel has been identified. We have also clarified the similarities and differences between the definitions based respectively on multiplicative cascades and on stochastic integrals.

We have also considered a $D$ dimensional generalization, showing that the simplification arising in the 1D case for stable random measures can also be introduced in $D$-dimension, using cylindrical coordinates. We have provided explicit expressions, using an integration over a $D$-dimensional space, with a kernel whose general expression was provided. We have first given the $2 D$ case before generalizing straightforwardly to $D \geq 2$. In each case we have also considered the spatio-temporal situation where $\mathbf{X}=(\mathbf{x}, t)$, which is built using the same kernel as for the purely spatial situation; only the integration domain is different, to ensure causality.

A general formulation in terms of a stochastic integral with respect to a Lévy noise allowed us to make clear the fundamental properties of such fields: multifractality both in time and space, homogeneity, stationarity and causality. The extension to the framework of generalized scale invariance as in [36] is considered. Such a time dependent positive valued process $\tilde{Q}_{\ell}(\mathbf{x}, t)$ exhibits a multifractal behaviour both in time and space. Moreover, it can be considered as more realistic than the approach proposed in [33-35], since in these works, the same quantities control both spatial correlations over small distances and temporal correlations over large time intervals. We emphasize that this is not the case in our definition and that the correlation function receives a quite intuitive geometrical interpretation (see fig. 6).

Finally, an important remark concerns the simulation of log-stable multiplicative cascades. They are usually rather painful to simulate because they do not belong to the family of compound Poisson cascades and cannot be obtained from a simple marked point Poisson process. The present results provide a much more simple way to the simulation of very similar $\alpha$-stable processes. The framework presented here corresponds to $D$-dimensional scalar multifractal processes. This is yet useful for practical applications. However this cannot be directly used for the simulation of real flows, since turbulence is fully tensorial. For real flow situations, one would need to generalize this continuous scalar framework to continuous multifractal tensorial processes. The corresponding theoretical framework is still to be developed. If turbulent cascade models could be adapted to vectorial or tensorial frameworks, it could lead to much more reliable predictive models for in- 


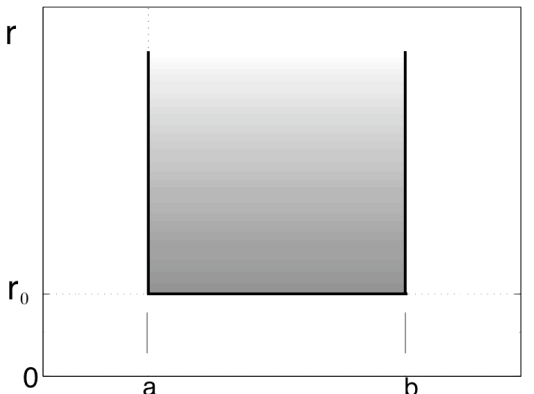

Fig. 7. Vertical plate denoted $T\left(r_{0}, a, b\right)$ in the text, in the $(t, r)$ space.

dustrial flows, than the eddy-viscosity models, which are usually only able to correctly predict moments of order 1 and 2 .

\section{A Stability property for random measures on thin strips: the 1D case}

This appendix is aimed at giving a hint to the computations used to link the stable stochastic measure of some set to some sotchastic integral with respect to some stable noise in dimension 1 .

We consider here a simple case, illustrated by Figure 7. We denote this set for $b>a, T\left(r_{0}, a, b\right)=\left\{\left(t^{\prime}, r^{\prime}\right): a \leq\right.$ $\left.t^{\prime} \leq b ; r_{0} \leq r^{\prime}\right\}$. We have $m\left(T\left(r_{0}, a, b\right)\right)=(b-a) / r_{0}$. And we consider here a Gaussian process with $\rho(q)=-\frac{1}{2} q^{2}$, so that:

$$
\mathbb{E}\left[\exp \left[q M\left(T\left(r_{0}, a, b\right)\right)\right]\right]=\exp \left[q^{2}(b-a) / 2 r_{0}\right]
$$

Let us note here $\omega$ a Gaussian random measure of variance $\sigma$ and integrated on an interval $(a, b)$ :

$$
\omega=W(\sigma, a, b)=\sigma \int_{a}^{b} d B(t)
$$

We then have:

$$
\mathbb{E}[\exp [q \omega]]=\exp \left[\frac{q^{2}}{2} \int_{a}^{b} \sigma^{2} d t\right]=\exp \left[q^{2}(b-a) \sigma^{2} / 2\right]
$$

Comparing equations (35) and (37), we see that the stable random variable $M\left(T\left(r_{0}, a, b\right)\right)$, which is a random measure on a $2 \mathrm{D}$ space, is then equal in distribution to a $1 \mathrm{D}$ process, following the stability property. This gives the following relation for a Gaussian random measure:

$$
M\left(T\left(r_{0}, a, b\right)\right) \triangleq W\left(r_{0}^{-1 / 2}, a, b\right)
$$

where $\triangleq$ means "equality in distribution". Applying this relation to the thin plate $T\left(r_{0}, t, t+d t\right)$ gives the following:

$$
M\left(T\left(r_{0}, t, t+d t\right)\right) \triangleq r_{0}^{-1 / 2} d B(t)
$$

For the stable case of basic index $0 \leq \alpha \leq 2$, we get:

$$
M\left(T\left(r_{0}, t, t+d t\right)\right) \triangleq r_{0}^{-1 / \alpha} d L_{\alpha}(t)
$$

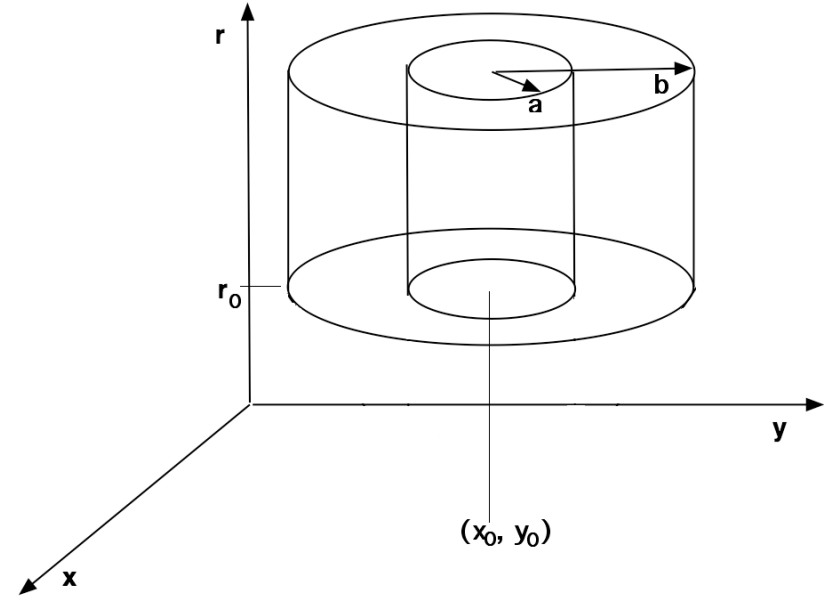

Fig. 8. Void cylinder denoted $V C\left(r_{0}, \mathbf{X}_{0}, a, b\right)$ in the text, in the $(x, y, r)$ space.

\section{B Stability property for random measures on a cylindrical corona: the $2 \mathrm{D}$ case}

We consider here the case of a void cylinder, illustrated by Figure 8. We denote this set for $b>a, V C\left(r_{0}, \mathbf{X}_{0}, a, b\right)=$ $\left\{\left(\mathbf{X}^{\prime}, r^{\prime}\right): a \leq\left\|\mathbf{X}^{\prime}-\mathbf{X}_{0}\right\| \leq b ; r_{0} \leq r^{\prime}\right\}$. On one hand, the measure of this set is given by:

$$
\begin{aligned}
m\left(V C\left(r_{0}, \mathbf{X}_{0}, a, b\right)\right) & =C(2) \pi\left(b^{2}-a^{2}\right) \int_{r_{0}}^{\infty} \frac{d r}{r^{3}} \\
& =\pi \frac{b^{2}-a^{2}}{2 r_{0}^{2}} C(2) .
\end{aligned}
$$

where $C(2)$ is the normalizing constant in 2 dimensions.

We consider here a Gaussian process $M\left(V C\left(r_{0}, \mathbf{X}_{0}, a, b\right)\right)$ depending on $\mathbf{X}_{0}$ and such that

$$
\log \mathbb{E}\left[\exp \left[q M\left(V C\left(r_{0}, \mathbf{X}_{0}, a, b\right)\right)\right]\right]=q^{2} \pi \frac{b^{2}-a^{2}}{4 r_{0}^{2}} C(2)
$$

On the other hand, let us note here $\omega$ a Gaussian random measure of variance $\sigma$ and integrated over a cylindrical corona of inner radius $a$ and outer radius $b$, centered at point $\mathbf{X}_{0}: \omega=\sigma \int_{A} d B(x, y)$, with $A\left(\mathbf{X}_{0}, a, b\right)=\left\{\mathbf{X}^{\prime}: a \leq\right.$ $\left.\left\|\mathbf{X}^{\prime}-\mathbf{X}_{0}\right\| \leq b\right\}$. We have:

$$
\log \mathbb{E}[\exp [q \omega]]=\sigma^{2} q^{2} \pi \frac{b^{2}-a^{2}}{2} C(2)
$$

Comparing equations (42) and (43), we see that the random variable $M\left(V C\left(r_{0}, \mathbf{X}_{0}, a, b\right)\right)$, which is a random measure on a $3 \mathrm{D}$ volume, is then equal in distribution to a random measure on a $2 \mathrm{D}$ surface, with variance $\sigma=$ $\left(\sqrt{2} r_{0}\right)^{-1}$. This gives the following relation:

$$
M\left(V C\left(r_{0}, \mathbf{X}_{0}, a, b\right)\right) \triangleq\left(\sqrt{2} r_{0}\right)^{-1} M\left(A\left(\mathbf{X}_{0}, a, b\right)\right)
$$

For the stable case of basic index $0 \leq \alpha \leq 2$, we have:

$$
M\left(V C\left(r_{0}, \mathbf{X}_{0}, a, b\right)\right) \triangleq\left(2 r_{0}^{2}\right)^{-1 / \alpha} M\left(A\left(\mathbf{X}_{0}, a, b\right)\right)
$$




\section{Stability property for random measures on hypercylindrical coronas: the D-dimensional case}

The previous approach for dimension 2 can be generalized for $D$ dimension. In this case, the only difference is the introduction of the term $r^{-D-1}$ instead of $r^{-3}$ in the integral of eq. (41) We still consider a void cylinder $V C\left(r_{0}, \mathbf{X}_{0}, a, b\right)=\left\{\left(\mathbf{X}^{\prime}, r^{\prime}\right): a \leq\left\|\mathbf{X}^{\prime}-\mathbf{X}_{0}\right\| \leq b ; r_{0} \leq r^{\prime}\right\}$ and provide here directly the stable case of index $\alpha$ :

$$
M\left(V C\left(r_{0}, \mathbf{X}_{0}, a, b\right)\right) \triangleq\left(D r_{0}^{D}\right)^{1 / \alpha} M\left(A\left(\mathbf{X}_{0}, a, b\right)\right)
$$

The author gratefully acknowledge Nicolas Perpete for stimulating discussions and comments.

\section{References}

1. U. Frisch, Turbulence; the legacy of A. N. Kolmogorov (Cambridge University Press, Cambridge, 1995) 296 p.

2. D. Schertzer, et al., Fractals 5, 427 (1997).

3. D. Schertzer, S. Lovejoy, J. Geophys. Res. 92, 9693 (1987).

4. P. Hubert, Hydrol. Sci. J. 46, 897 (2001).

5. L. Seuront et al., J. Plankton Res. 21, 877 (1999).

6. S. Lovejoy et al., J. Plankton Res. 23, 117 (2001).

7. F. G. Schmitt, L. Seuront, Physica A 301, 375 (2001).

8. Y. Ashkenazy et al., Physica A 316, 662 (2002).

9. E. Wesfreid et al., Appl. Comput. Harmon. Anal. 18, 329 (2005).

10. I. J. Benczik, Z. Neufeld, T. Tel, Phys. Rev. E 71, 016208 (2005).

11. E. Marsch, C.-Y. Tu, H. Rosenbauer, Ann. Geophysicae 14, 259 (1996).

12. F.G. Schmitt, D. Schertzer, S. Lovejoy, Appl. Stoch. Models Data Anal. 15, 29 (1999).

13. J.-F. Muzy, D. Sornette, J. Delour, A. Arneodo, Quant. Finance 1, 131 (2001).

14. L. Calvet, A. Fisher, J. Econometrics 105, 27 (2001).

15. B. Castaing, B. Dubrulle, J. Phys. II France 5, 895 (1995).

16. P. Chainais, Eur. Phys. J. B 51, 229 (2006).

17. P. Chainais, IEEE Trans. on Pattern Analysis and Machine intelligence, in press (2007).

18. B. Mandelbrot, J. Fluid Mech. 62, 305 (1974).

19. A. Arneodo, E. Bacry, J.-F. Muzy, J. Math. Phys. 39, 4142 (1998).

20. F. G. Schmitt, D. Marsan, Eur. Phys. J. B 20, 3 (2001).

21. J.-F. Muzy, E. Bacry, Phys. Rev. E 66, 056121 (2002).

22. F. G. Schmitt, Eur. Phys. J. B 34, 85 (2003).

23. E. Bacry, J.-F. Muzy, Comm. Math. Phys. 236, 449 (2003).

24. P. Chainais, R. Riedi, P. Abry, IEEE Trans. Infor. Theor. 51, 1063 (2005).

25. B. Rajput, J. Rosinski, Probab. Theory Relat. Fields 82, 451 (1989).

26. G. Samorodnitsky and M. S. Taqqu,Stable Non-Gaussian Random Processes; Stochastic Models with Infinite Variance (Chapman Hall/CRC, London, 1994) 632 pp.

27. D. Applebaum,Lévy Processes and Stochastic Calculus (Cambridge University Press, Cambridge, 2004) 384 pp.

28. W. Feller, An Introduction to Probability Theory and its Applications, vol. II, 2nd edn. (Wiley, New York 1971).
29. J. Barral, B. Mandelbrot, Probab. Theory Relat. Fields 124, 409 (2002).

30. P. Chainais, R. Riedi, P. Abry, Traitement du Signal 22, 1 (2005).

31. B. Lashermes, P. Abry, P. Chainais, Int. J. Wavelets, Multires. and Info. Proc. 2, 497 (2004).

32. S. Kida, J. Phys. Soc. Japan 60, 5 (1991).

33. O. E. Barndorff-Nielsen, J. Schmiegel, Uspekhi Mat. Nauk. 159, 63 (2003).

34. J. Schmiegel, J. Cleve, H. C. Eggers, B. Pearson, M. Greiner, Phys. Lett. A 320, 247 (2004).

35. J. Schmiegel, O. E. Barndorff-Nielsen, H. C. Eggers, South African J. Sci. 101, 513 (2005).

36. D. Marsan, D. Schertzer, S. Lovejoy, J. Geoph. Res., 101(D21) (1996).

37. S. Lovejoy, D. Schertzer, Wat. Resour. Res., 21, 1233 (1985).

38. S. Pecknold, S. Lovejoy, D. Schertzer, C. Hooge, J.-F. Malouin, Cellular Automata: Prospects in astrophysical applications, edited by J.M. Perdang and A. Lejeune, pp. 228-267 (World Scientific, 1993). 